MEASURING THE EFFECT OF A COMMUNITY RESPIRATORY SERVICE ON CHRONIC OBSTRUCTIVE PULMONARY DISEASE (COPD) EMERGENCY ADMISSIONS TO HOSPITAL: A CONTROLLED INTERRUPTED TIME SERIES ANALYSIS

${ }^{1}$ KA Levin*, ${ }^{2} \mathrm{M}$ Milligan, ${ }^{1} \mathrm{E}$ Crighton, ${ }^{3} \mathrm{D}$ Anderson. ${ }^{1}$ Public Health Directorate, NHS GGC, Glasgow, UK; ${ }^{2}$ Community Respiratory Team, NHS GGC, Glasgow, UK; ${ }^{3}$ Emergency Care and Medical Services, NHS GGC, Glasgow, UK

\subsection{6/jech-2019-SSMabstracts.73}

Background A community respiratory service was implemented in the North West of Glasgow in January 2013, as part of the Reshaping Care for Older People Programme (RCOPP). The service comprises a team of physiotherapists, occupational therapists and support workers to work with older COPD patients, providing education and self-management advice, and, where appropriate, treatment at home, in order to reduce the risk of hospital admission. This study aimed to measure the impact of the service on emergency admissions (EAs) to hospital due to COPD.

Methods COPD EAs were defined as emergency admissions to hospital with a primary diagnosis of COPD. Rate of COPD EAs per 1000 population aged 65 years + in Glasgow City was compared before and after onset of the service with a 10 month phase-in period, using segmented linear regression with 21-month (April 2011- December 2012) pre- and 17-month (November 2013-March 2015) post- intervention periods. Rate of COPD EAs for residents of South and North East Glasgow $(\mathrm{S}+\mathrm{NE})$ - areas within Glasgow City, but with no such service in place - were used as a comparison group. The model adjusted for the rate of all-cause emergency admissions in order to control for the effect of other initiatives set up during the RCOPP. Autoregressive and moving average terms were included in the model, as well as a fourier term to adjust for seasonality. Results Comparing April-July 2011 and April-July 2012, rate of COPD EAs increased in NW but remained approximately the same in S+NE. Rates reduced in both areas by 2014. By comparison, all-cause EAs rose in NW before 2013, followed by a reduction thereafter while in $\mathrm{S}+\mathrm{NE}$ reductions were seen in each consecutive year. Adjusting for all cause EAs and changes in $\mathrm{S}+\mathrm{NE}$, and therefore factoring out the impact of other RCOPP initiatives, the impact of the service was found to be a level change of $-0.28(-0.32,-0.24)$ and a trend change of $-0.02(-0.02,-0.01)$ COPD EAs per 1000 per month. This is equivalent to a predicted reduction due to the service of -0.57 COPD EAs per 1000 popn per month, in March 2015, and a relative reduction of $24.7 \%$. Rate of COPD EAs per month reduced over time after the introduction of the service (from the point of full staffing).

Conclusion After factoring out the impact of other possible RCOPP initiatives, the community respiratory service was associated with a significant reduction in the rate of COPD EAs.

\section{OP71 PATIENT CHOICE AND EQUALITY OF ACCESS IN SCOTLAND: AN ANALYSIS OF NHS FUNDED TREATMENTS IN THE NHS AND PRIVATE SECTOR}

G Kirkwood* , AM Pollock. Institute of Health and Society, Newcastle University, Newcastle, UK

10.1136/jech-2019-SSMabstracts.74

Background In February 2003, 'Partnership for Care - Scotland's Health White Paper' invoked spare capacity in the private sector as a means of treating NHS patients whose waiting times exceeded the national guaranteed limits. No evidence was given in support of the policy of giving patients a choice of provider with the intention that this would 'complement and not detract from NHS Boards' corporate responsibility to develop sustainable local solutions to long waits'.

Previous research using data on elective hip replacements has found evidence that the use of the private sector to provide NHS funded treatment disadvantaged older patients and patients from the most socio-economically deprived areas of Scotland.

Methods Data on NHS funded elective operations performed by the NHS and by private providers were extracted from Information Services Division NHS Scotland admitted patient care datasets for: hip replacements; knee replacements; cataract operations; arthroscopies; cholecystectomies; and inguinal hernia operations.

Using a segmented regression model built around the introduction of patient choice, changes in inequality between patients living in the most socioeconomically deprived and least socioeconomically deprived areas of Scotland were analysed. Similarly models were built to test for changes in inequality between the oldest patients and the other age groups.

Results Initial findings show that after the introduction of patient choice, there is evidence that patients living in the most socio-economically deprived areas had levels of inequality increase for cataract operations, $1.62 \%$ increase $(95 \%$ confidence interval $1.58 \%, 1.66 \%$ ) and cholecystectomies, $0.42 \%$ increase $(0.36 \%, 0.48 \%)$ although inequality may have decreased by $0.66 \%(0.64 \%, 0.68 \%)$ for arthroscopies.

For the oldest patients (85 years and over for hip, knee and cataract; 70 years plus for arthroscopy; and 80 years plus for cholecystectomies; and inguinal hernia operations) there is evidence of improving inequality for hip replacements with a $1.42 \%$ reduction in inequality $(1.53 \%, 1.31 \%)$ whereas inequality appears to have increased by $0.11 \% \quad(0.09 \%$, $0.13 \%)$ for cataract patients and by $4.00 \%$ (3.96\%, 4.03\%) for arthroscopy patients.

Conclusion Patient choice was introduced in Scotland and England with no evidence to support it and with very little evaluation since. This research shows that that for some of the most vulnerable patients and for certain procedures there may be a widening of inequalities which may be a result of the operation of patient choice. Conversely reductions in inequality may have occurred in some cases. Further research is necessary to understand the mechanisms involved in any such changes in inequality.

\section{OP72 EFFECTIVENESS OF PRIMARY-CARE BASED COMMUNITY-LINKS PRACTITIONERS IN AREAS OF HIGH SOCIOECONOMIC DEPRIVATION}

${ }^{1} S$ Wyke*, ${ }^{1} B$ Fitzpatrick, ${ }^{1} \mathrm{~L}$ Grant, ${ }^{1} \mathrm{~N} R$ Chng, ${ }^{1} \mathrm{~A}$ McConachie, ${ }^{1} \mathrm{~A}$ Bakhshi, ${ }^{1} \mathrm{G}$ James-Rae, ${ }^{1} \mathrm{C}$ O'Donnell, ${ }^{2} S$ Mercer. 'Institute of Health and Wellbeing, University of Glasgow, Glasgow, UK; ${ }^{2}$ Centre for Population Health Sciences, University of Edinburgh, Edinburgh, UK

\subsection{6/jech-2019-SSMabstracts.75}

Background A common policy response to health inequalities is the introduction of social prescribing programmes. Social prescribing aims to link patients to non-medical sources of support within a community, expanding options and resources 
beyond those traditionally provided in primary healthcare. Providing access to community-based services is expected to, for example, help reduce social isolation, provide access to initiatives supporting behaviour change (such as walking groups) and mitigate some of the effects of poverty by access to welfare advice or employment opportunities. Although widespread, the evidence-base for the effectiveness of social prescribing is extremely limited. We aimed to assess the effect of a form of social prescribing, the primary care-based community links practitioner (CLP) programme, on patients' quality of life and wellbeing.

Methods Quasi-experimental cluster randomised controlled trial in socioeconomically deprived areas of Glasgow, Scotland. Adult patients ( $\geq 18$ years) referred to CLPs in seven intervention practices were compared with a random sample of adult patients from eight comparison practices at baseline and 9 months. Primary outcome; health-related quality of life (EQ5D-5L). Secondary outcomes; wellbeing (ICE-CAP A), depression (HADS-D) anxiety (HADS-A), and self-reported exercise. Multilevel, multi-regression analyses adjusted for baseline differences. Patients were not blind to the intervention, but outcome analysis was masked.

Results Data were collected on 288 and 214 (72.4\%) patients in the intervention practices at baseline and follow-up, and on 612 and 561 (92\%) patients in the comparison practices. Intention to treat analysis found no differences between the two groups for any outcome. In sub-group analysis, patients who saw the CLP on three or more occasions (45\% of those referred) had significant improvements in EQ-5D-5L, HADSD, HADS-A and exercise levels. There was a high positive correlation between CLP consultation rates and patient uptake of suggested community resources

Conclusion We were unable to prove the effectiveness of referral to CLPs based in primary care in deprived areas on improving patient outcomes. Future efforts to boost uptake and engagement might improve overall outcomes, although the apparent improvements in those who regularly saw the CLPs may be due to reverse causality. Further research is required before wide-scale deployment of this approach.

\section{Pregnancy/Maternal Health 1}

\section{OP73 THE IMPACT OF CHRONIC HYPERTENSION ON ADVERSE MATERNAL AND PERINATAL OUTCOMES: A SYSTEMATIC REVIEW AND META-ANALYSIS}

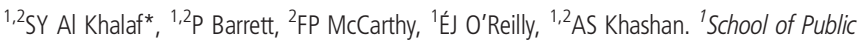
Health, University College Cork, Cork, Ireland; ${ }^{2}$ The Irish Centre for Fetal and Neonatal Translational Research (INFANT), University Maternity Hospital, Cork, Ireland

10.1136/jech-2019-SSMabstracts.76

Background Chronic hypertension affects up to $5 \%$ of all pregnancies, and this is expected to rise due to increasing prevalence of maternal obesity. This study aimed to systematically review observational studies to investigate the risk of adverse perinatal outcomes among pregnant women with chronic hypertension compared with normotensive women.

Methods Medline/PubMed, EMBASE, and Web of Science were searched (from first publication until 10th January 2019) to identify peer-reviewed articles without restriction on language or study period. We included observational studies based on the following criteria: 1) participants were pregnant women; 2) exposure was chronic hypertension; 3) comparison was normotensive women; 4) outcomes included measures of at least one of the following: superimposed pre-eclampsia, small for gestational age, stillbirth, preterm birth, caesarean section, neonatal intensive care unit admission, low birth weight, post-partum hemorrhage maternal death and neonatal death. This review is registered in PROSPERO (CRD42019120088).

Two investigators independently reviewed the eligibility criteria, extracted the data and assessed the quality of included studies using the Newcastle-Ottawa tool. A meta-analysis was performed using RevMan 5.3 for each exposure-outcome association, when data allowed. Random effect models were applied for pooling crude and adjusted odds ratios (ORs) respectively. Heterogeneity among studies was assessed using a Cochrane $\mathrm{Q}$ statistic and the Higgins $\mathrm{I}^{2}$ test. Sensitivity analysis was performed by study design, study location, decade of publication, and according to study quality. Publication bias was assessed using Begg's funnel plot and Egger's test. The effect of using antihypertensive medications on the risk of adverse maternal and perinatal outcomes will also be analysed as part of this review.

Results Of the 9739 articles identified, 69 studies met the inclusion criteria. Thirteen studies reported adjusted estimates for small for gestational age (including 7,070,558 participants); adjusted pooled $\mathrm{OR}=1.97$ (95\% CI, 1.46, 2.67) among women with chronic hypertension compared to normotensive. Similarly, eleven studies reported adjusted estimates for stillbirth (including 15,231,939 participants), with a pooled adjusted $\mathrm{OR}=2.36$ (95\%CI, 2.18, 2.55). Four studies reported adjusted estimates for neonatal death, the adjusted pooled $\mathrm{OR}=2.29$ (95\%CI, 2.03, 2.60). The analyses of other outcomes are ongoing.

Conclusion There are strong associations between chronic hypertension and adverse perinatal outcomes, including small for gestational age, stillbirth and neonatal death. Most studies in this review did not take severity of hypertension into account when comparing the outcomes. This review summarises current knowledge on the association between chronic hypertension and adverse perinatal outcomes and may be used to optimise antenatal care and pregnancy outcomes.

\section{OP74 MATERNAL SMOKING DURING PREGNANCY AND RISK OF TYPE 1 DIABETES: WHOLE-OF-POPULATION STUDY}

${ }^{1,2} \mathrm{M}$ Begum* ${ }^{*}{ }^{1,2} \mathrm{RM}$ Pilkington, ${ }^{1,2} \mathrm{CR}$ Chittleborough, ${ }^{1,2,3} \mathrm{JW}$ Lynch, ${ }^{1,2,4} \mathrm{M}$ Penno, LG Smithers. "School of Public Health, The University of Adelaide, Adelaide, Australia; ${ }^{2}$ Robinson Research Institute, The University of Adelaide, Adelaide, Australia; ${ }^{3}$ Population Health Sciences, University of Bristol, Bristol, UK; ${ }^{4}$ Adelaide Medical School, The University of Adelaide, Adelaide, Australia

10.1136/jech-2019-SSMabstracts.77

Background Evidence about maternal smoking during pregnancy and type 1 diabetes (T1D) risk is inconsistent. Most studies have small numbers of children exposed to prenatal smoking, and some were unable to look at timing of exposure to smoking, or were at risk of bias due to unmeasured confounding. Therefore, the objectives of this study were: 1) to estimate the association between prenatal smoking and T1D risk, looking at the timing of exposure to smoking (throughout pregnancy, first-half, or second-half of pregnancy), with adjustment for a range of confounding factors defined a priori; 2) to perform a negative-control outcome analysis to 